\title{
Investigaciones radiofónicas: de la radio a la radio indígena. Una revisión en Colombia y Latinoamérica*
}

\author{
Óscar Julián Cuesta Moreno
}

Recibido: 20 de febrero de 2012 Aprobado: 27 de marzo de 2012

\begin{abstract}
Resumen
El artículo expone los resultados de una revisión documental sobre el tema de la radio indígena en Colombia. Para ello, en primer lugar, presenta las publicaciones sobre radio en el país desde 1974 en adelante. En un segundo momento, observa las indagaciones realizadas en la relación medios de comunicación-indígenas. Al ser el tema de la radio indígena una temática poco explorada en Colombia, muestra una recopilación de pesquisas en esta materia en América Latina. Finalmente, da a conocer las investigaciones colombianas en esta línea y analiza las tensiones legales de su eclosión. Concluye argumentando la necesidad de ampliar las iniciativas sobre radio indígena y propone observar cómo la cosmovisión de estos pueblos re-significa el lenguaje y los formatos radiofónicos modernos.
\end{abstract}

Palabras clave: radio indígena, lenguaje radiofónico, cosmovisión, producción radiofónica, cultura.

* Este artículo es resultado de la revisión realizada en el marco del proyecto La cosmovisión en la re-significación de la producción radiofónica de las emisoras indígenas, del grupo de investigación Comunicación, cultura y tecnología, financiado por la Fundación Universitaria Los Libertadores.

** Magíster en Educación de la Universidad Pedagógica Nacional. Especialista en Docencia Universitaria de la Universidad Cooperativa de Colombia. Comunicador Social de la Universidad Santo Tomás De Aquino y Técnico en Locución y Medios Audiovisuales del Colegio Superior de Telecomunicaciones.Correo electrónico: oscarcuesta@colombia.com 


\title{
Radio Research: From Radio to Aborigine Radio. A Review in Colombia and Latin America
}

\begin{abstract}
This article shows the results of a documentary revision about the aborigine radio in Colombia. For this purpose, the article initially shows publications about radio in the country since 1974. Then, it shows the research conducted associated to aborigine communication media. Since the topic on aborigine radio has been poorly explored in Colombia, the article shows a number of investigations conducted in this field in Latin America. Finally, the article reveals Colombian research in this field and analyzes legal consequences of its emergence. The article concludes by posing the need for widening the initiatives with respect to aborigine radio and proposes to observe how world vision of these populations gives a new meaning to language and modern radio formats.
\end{abstract}

Key words: Aborigine radio; radio language; world vision; radio production; culture. 


\section{Introducción}

El reconocimiento de los saberes y prácticas culturales de las comunidades originarias de América es una preocupación cada vez más reiterada en las nuevas dinámicas de intercambio global. Este reconocimiento, que tiene amplia discusión en el sector educativo bajo la lógica intercultural, se manifiesta en escenarios mediáticos organizados por estrategias comunicativas de tipo alternativo, como es el caso de las emisoras indígenas en Colombia.

El presente artículo hace una revisión documental de la literatura sobre radios indígenas en Colombia, procurando rastrear las investigaciones y opiniones en libros, artículos y ponencias que, de alguna manera, han reflexionado la manifestación cultural mediante la producción radiofónica de estas emisoras.

Si bien el artículo tiene como propósito central mostrar la revisión del tema de la radio indígena en Colombia (incluyendo los referentes legales que permiten su eclosión), también pretende dar cuenta de los trabajos que han abordado la radio en el país, la relación medio-indígena e investigaciones sobre radio indígena en otros países latinoamericanos.

\section{Metodología}

La presente revisión tuvo como criterio el rastreo de publicaciones desde 1974, año en que se publica el primer libro que da cuenta de la historia de la radio en Colombia, al 2011. Hay que decir, de antemano, que la investigación y producción literaria sobre el tema radiofónico en Colombia es corta y, mucho más, sobre radio indígena.

La metodología respondió a un enfoque cualitativo de corte interpretativo. La categoría principal de análisis fue radio indígena, sin embargo, a lo largo del proceso de búsqueda se observó la necesidad de ampliar el corte categorial e incluir otras, como la de comunicación indígena. A continuación se especifican los momentos de búsqueda:

La primera fase de esta revisión tuvo como eje central las indagaciones y publicaciones sobre radio en Colombia. Posteriormente, se profundizó la revisión sobre radio indígena en el país, así como sus marcos legales. Al observar las pocas investigaciones en esta materia, se buscaron trabajos sobre medios de comunicación e indígenas en Colombia. Finalmente, se amplió la búsqueda a experiencia de emisoras indígenas en Latinoamérica, para observar las preocupaciones investigativas en otras experiencias del continente. La estructura del artículo no responde a estas fases metodológicas, pues va dejar para lo último la revisión sobre radio indígena en el país, observando algunas similitudes de estas investigaciones con otras experiencias en el continente.

\section{Resultados y discusión}

Es importante citar en esta revisión, en primer lugar, las palabras de Valderrama (2009), quien asegura que los trabajos sobre radio en Colombia tienen, haciendo una comparación cuantitativa, una menor acogida: se investiga y publica más sobre televisión y, en segundo lugar, sobre prensa.

Del mismo modo, él afirma que en su indagación sobre el estado del arte de las investigaciones en medios de comunicación en Colombia que "las que menor frecuencia presentan (con menos de diez documentos asociados) son televisión regional, indígenas, mujer, salud, espacio público" (Valderrama, 2009, p. 269).

En esta misma línea, Rojas (2009, p.110) pregunta: "si no hay investigaciones y producción académica sobre la construcción de políticas públicas de la radio y la prensa, ¿̇sobre qué tipo de conocimiento descansan las normativas que sobre ellos se diseñan?". Y contínua: "Nos preguntamos: ¿por qué no se consideran las especificidades de 
la radio y la prensa de la misma manera como se defienden y argumentan las particularidades de la televisión?". Concluye que la ausencia de investigaciones sobre las políticas de radio y prensa no permite conocer la contribución de estos medios a los procesos de democratización, participación y libertad de expresión de los individuos y de las comunidades.

Siguiendo las anteriores ideas, se puede observar, en primer lugar, dos hechos que entrelazan el eje de esta revisión: por un lado la radio es el medio en Colombia del que menos trabajos se han realizado y, por otro lado, el tema indígena tiene poca frecuencia de asociación con investigaciones de medios de comunicación en el país. En consecuencia, se puede asegurar que una investigación sobre radios indígenas, como la que presenta este proyecto, tiene una inmediata pertinencia, pues estaría tocando límites poco explorados.

A lo anterior se suma que "Los movimientos indígenas de América y del resto del mundo han desarrollado diferentes acciones en la búsqueda del reconocimiento por parte de los Estados $\mathrm{Na}$ cionales de los derechos políticos, económicos, lingüísticos, sociales, culturales, religiosos. La Radiodifusión sonora es el medio de comunicación social que más ha logrado romper barreras de las lenguas dominantes" (Fuentemayor y Antepaz, 2009, p. 9).

\section{Trabajos sobre radio en Colombia}

Las publicaciones sobre radio en Colombia tienen referentes ya clásicos en lo que respecta a la historia del medio: Téllez (1974), y su libro Cincuenta años de la radiodifusión colombiana, Pareja (1984), que hace una revisión desde 1929 a 1980, y Múnera (1999) que amplía su análisis e involucra a la televisión. En 1992, Gil analiza la influencia política y del poder económico en las cadenas radiofónicas colombianas. Él afirma que se dio una "paulatina concentración de las emisoras en manos de unos pocos conglomerados económicos, dueños, asimismo, de empresas de otros sectores industriales" (Gil, 1992, p. 254).

Urdaneta (1994) hace una revisión específica de la radiodifusión en Barraquilla, ciudad que vio nacer el medio en el país. Otras investigaciones a finales de la última década del siglo XX abordaron el tema de la radio en el país; se destaca el caso de Lalinde, quien publica tres trabajos: en 1999, observó el tema de género en el medio; en 1998, publica un artículo analizando la participación de los oyentes en las dinámicas radiales de la época, y en 1992 presenta una investigación de la dinámica profesional en la producción de noticieros radiales, y toma por caso la producción de Caracol. Siguiendo con esta cadena radial, se destaca la publicación de Pérez (1998), quien hace un recorrido histórico de la cadena y reflexiona sobre el papel y las dinámicas radiales en el tercer milenio.

Más recientemente, Castellanos (2001) publicó un artículo reflexivo sobre la radio con el propósito de hacer un balance historiográfico sobre las décadas del 30 al 50 del siglo pasado con el fin de incentivar nuevas preguntas para futuras investigaciones en el campo radial colombiano, entre ellas, la influencia del medio en el proceso de secularización, el papel de la radio comercial y su influencia en el proyecto de nación y rastrear las iniciativas del Estado sobre políticas culturales que ven a los medios no solo como transmisores sino como formadores de cultura. Siguiendo una preocupación similar a esta última, años antes, López de la Roche (1999) discutió la necesidad de comprender los medios de comunicación como procesos insertos en los sistemas culturales de la sociedad y, por ello, la importancia de incluirlos en la elaboración de políticas públicas sobre cultura.

Antequera y Obregón (2002) presentan una investigación que da cuenta de la radio como dinamizadora de procesos sociales y culturales en Barranquilla. La indagación observó cómo en esta ciudad el medio se ha empezado acercar a la comunidad para entablar diálogo sobre sus 
problemáticas e inquietudes: "esto se materializa en casos como el apoyo a grupos folclóricos, la apertura de espacios de denuncia ciudadana sobre irregularidades en el sector público y privado (como el caso del incremento ilegal de pensiones escolares), el acceso a una mayor pluralidad religiosa, y el acercamiento a instituciones del orden civil y de justicia" (Antequera y Obregón, 2002, p. 148).

En el plano de las emisoras comunitarias, Saffon y Uprimny (2007) analizan las luchas de las comunidades de Bogotá por el derecho a crear emisoras comunitarias en las ciudades capitales, pues tal como está la legislación, no es posible. Estos autores aseguran que "las emisoras comunitarias servirían como mecanismo de empoderamiento de organizaciones locales de base y de fortalecimiento de los lazos sociales y del sentimiento de pertenencia, identidad y solidaridad de las comunidades marginales, todo lo cual enriquecería enormemente la democracia" (Saffon y Uprimny, 2007, p. 223). Siguiendo con radio comunitaria, el Ministerio de Comunicación (2002) divulgó un diagnóstico del servicio comunitario de de radiodifusión sonora en Colombia; en él se plantea que la mayoría de estas emisoras nacen por la necesidad de abrir espacios fuera de los comerciales.

En los últimos años, sobre la producción radial en Colombia se pueden citar dos trabajos: Rocha y otros (2010) quienes analizan el papel de la radio comunitaria como dinamizadora de los procesos participativos basados en una investigación desarrollada en once municipios de Cundinamarca. En el 201 1, Barrios publica un libro donde hace un paralelo entre la radio convencional y la radio virtual, y explica las diferencias técnicas y de producción entre una y otra.

Es pertinente referenciar el trabajo de Valderrama y Rojas (2007), quienes plantean el ejercicio de construir el estado del arte sobre la investigación en medios en Colombia desde 1980 a 2005 , con el propósito de sistematizar los trabajos en la materia e identificar las preguntas, y puestas teóricas y metodológicas de los mismos.

De trabajos académicos de pregrado, en el úl timo tiempo, se pueden destacar dos ejercicios: el de Ruiz (2009), que hace un recorrido por las manifestaciones religiosas y esotéricas en las emisoras de Bogotá y analiza su papel en la divulgación de estas creencias. Por otro lado, González (2009) hace un estudio de audiencias sobre la emisora Javeriana Estéreo; esta indagación se limitó a los mismos estudiantes de esta universidad.

\section{Algunos trabajos recientes sobre radio fuera de Colombia}

Igualmente, es pertinente observar otros trabajos de radio recientes en otros países. En primer lugar, se puede traer a colación la publicación de Winocur (2002), que aborda el tema de la construcción de lo público en la radio, a partir del concepto de ciudadano mediático (engloba medios, opinión pública y vida cotidiana) desde un análisis que hace con oyentes mexicanos. En el 2003, Lara y Vera centran su indagación sobre el paso de la radio análoga a la digital, especialmente, los aspectos técnicos.

Cebrián (2007) trabaja sobre modelos de radio en el marco de las innovaciones tecnológicas que permiten, más que el diálogo y participación, la interactividad, para lo cual analiza la convergencia de Internet y la telefonía móvil. Con respecto a Internet, el autor considera que posibilita la interculturalidad, pues la emisora deja de ser local para conversar con el mundo. Similar a este trabajo es el de Herrera (2007), que observa las nuevas modalidades para la participación de los jóvenes en la radio española. Recientemente, Rodríguez (2011) reflexiona sobre cómo los nativos digitales son oyentes con características específicas, pues nacieron cuando ya estaba en boga la tecnología digital, así que exigen una reconfiguración de la producción radiofónica, que implica, entre otras 
cosas, reconocer al oyente como productor y emisor de información, es decir, ya no un receptor pasivo.

Con respecto a la radio en procesos sociales o comunitarios, hay que destacar a Ilboudo y Castello (2004), quienes realizan una reflexión y propuesta de cómo utilizar la radio y otras tecnologías en los procesos rurales, para reforzar la identidad nacional y reducir las diferencias con las zonas urbanas.

Siguiendo con la radio en zonas rurales, Bennett (2004), en su trabajo sobre la Red Rusa de Información Rural, identifica estas cualidades para el medio:

Ningún otro medio tiene el potencial de la radio para crear condiciones y proporcionar a la gente un acceso genuino a información útil; a la vez permite expresar sus sentimientos, opiniones, puntos de vista, sueños y aspiraciones, miedos e inseguridades, fortalezas y capacidades y por supuesto, sus ideas. La radio es una herramienta útil para involucrar a las comunidades en procesos participativos y de consenso sobre las prioridades de desarrollo (Bennett, 2004, p. 128).

\section{Medios y comunidades indígenas}

Después de observar trabajos sobre radio en Colombia, la investigación limitó sus parámetros sobre la radio indígena en Latinoamérica. Antes de mostrar los resultados, es pertinente decir que existen trabajos que han abordado una categoría más amplia: la relación medios-comunidades indígenas.

Se puede citar, por ejemplo, la publicación de Albó (1998), quien realizó un trabajo sobre la expresión indígena y medios de comunicación; para el autor, que analiza el fenómeno en el marco de la diglosia, es decir, cuando en un región una lengua tiene preeminencia sobre otra, el análisis de la estructuras de poder es recurrente, pues al fin de cuentas el español se impone sobre las lenguas indígenas porque es el idioma de las clases gobernantes, los mestizos y los colonos.

Salazar (2002) afirma que, a pesar del uso alternativo de los medios, la tecnología sigue siendo un factor de desigualdad social que privilegia a las clases con mayores posibilidades económicas; además, hace un llamado a profundizar la investigación y teorización de los medios en los movimientos indígenas, resaltando la radio por sus características orales y su alcance geográfico.

Se puede destacar la indagación de Arteaga y González (2004) sobre los medios de comunicación y las identidades indígenas, que es desarrollada en el marco de la posibilidad de lo "otro", es decir, perspectivas, miradas, fuera de los parámetros y estereotipos occidentales. Otra indagación de estos investigadores se centra en la representación de lo indígena en los medios de comunicación, que examina cómo manejan los medios de comunicación la información sobre los pueblos indígenas en Colombia (González y Arteaga, 2005).

Las comunidades indígenas de Totoroes, Kokonuco, Yanacona, Guambiano y Nasa en convenio con la Escuela de Comunicación Social de la Universidad del Valle realizaron un monitoreo de medios del cual concluyeron que estos realizan las siguientes representaciones de lo indígena (MINGA, 2005):

- Colectivización: se presenta a lo indígena como unidad indiferenciada, es decir, todos son iguales, desconociendo lenguas, cosmovisiones, prácticas culturales, etc.

- Criminalización: las acciones de los movimientos indígenas son puestos como una disfunción social, como una amenaza al orden y la institucionalidad.

- Segregación y exclusión: demarca los límites identitarios, destaca las diferencias. Por ejem- 
plo, los derechos y tradiciones indígenas son resaltados como algo singular, fuera de los parámetros convencionales de la mayoría: "no son como nosotros", es la connotación.

- Cosificación: el otro es objeto de fuerzas de sujetos con dominio. No actúa, no tiene potencia, se mueve por la potestad del otro.

- Arcaización: indica que el tiempo de los indígenas es, o era, otro tiempo.

En esta misma dirección, es decir, sobre la representación de lo indígena en los medios, Otero (2007) realiza un análisis junto con representantes de cinco pueblos indígenas del Cauca y con la asesoría de la misma Escuela de la Universidad del Valle. En palabras de este autor "El monitoreo estaba encaminado a servir de instrumento para trabajar, con periodistas y medios, una política de información no racista, más incluyente y respetuosa de las diversidades sociales y culturales que nos constituyen como nación de un país pluralista y democrático" (Otero, 2007, p.1).

Un referente importante en el uso de medios en los movimientos indígenas es el Consejo Regional Indígena del Cauca (CRIC), que tiene un importante protagonismo en el fortalecimiento de la Asociación de Medios Indígenas de Colombia (Red AMCIC). Según Otero (2008, p. 11) la organización fue "creada a finales de la década de los noventa con el objeto de fortalecer colectivos de comunicación, especialmente el proyecto de radio indígena, que promueve la difusión de identidades locales, sus conceptos de vida y la convivencia, asumiendo la diversidad cultural como la riqueza más importante del Cauca y Colombia".

Alí (2011) realiza un análisis de los medios de comunicación, los asuntos étnicos y las apuestas interculturales. Sobre la radio indígena afirma que "Se trata, en la mayoría de los casos, de radios comunitarias de débil potencia cuyo alcance, normalmente, no consigue sobrepasar los límites de los resguardos en los cuales operan" (Alí, 2011, p. 7).

\section{Radios indígenas en América Latina}

Posteriormente a observar estas investigaciones sobre la relación medios-indígenas, se presentan a continuación los trabajos sobre radios indígenas en Latinoamérica.

Iriarte (2006) hace un trabajo sobre una radio indígena comunitaria en Brasil, para lo cual centra su análisis en la emisora Pataxó-Hã-hã-hãe. Esta investigación tuvo el apoyo de la Oficina Regional de la UNESCO de Comunicación e Información para América Latina y el Caribe (ORCIALC) y, de manera preponderante, sus resultados resaltan el papel de esta emisora en las dinámicas de unidad comunitaria y las prácticas de una cultura de paz. En este trabajo se puede leer cierta perspectiva teórica de Gumucio (2004), pues recupera las experiencias comunitarias, reconoce el papel de los actores locales, rechaza la información vertical y recupera el desarrollo desde agendas propias.

En el plano de la interculturalidad, Fuentemayor y Antepaz (2009) realizan una investigación sobre las producciones radiofónicas bilingües en Zulia, Venezuela. Una de sus conclusiones es que no es suficiente crear radios que tengan programación bilingüe, en este caso el idioma Wuayuu (wayuunaiki) y español, para hacer un ejercicio profundo de intercambio cultural, sino que las emisoras deben tener una agenda, estructura y funcionamiento particular que responda a esos propósitos y permita el intercambio cultural entre los indígenas y los Alijunas (personas no Wayuu) ${ }^{1}$. Yendo más allá, aseguran que "La comunicación intercultural radiofónica debe ser realizada por y con la visión de los indígenas" (Fuentemayor y Antepaz, 2009, p.25).

1 En la sistematización de esta experiencia los autores citan los programas que desarrolla una de estas emisoras, aquí sus nombres: Jalian Wayuuirua (Adelante Guajiros), Alatakaa Sumuin Wayuu (Acontecer Indígena), Putchimaajachi (El portador de palabras) y No'upaluu Wayuu (Visión Indígena), programas radiales desde la perspectiva, filosofía y el compromiso indígena Wayuu. 
Igualmente, el anterior trabajo hace hincapié en la importancia de la Ley de Idiomas Indígenas, promulgada el 20 de mayo de 2008 en Venezuela, pues abre el espacio de las lenguas indígenas y su identidad cultural en los medios de comunicación. Los autores hablan de la lengua indígena como un elemento de transmisor cultural y los medios electrónicos como plataformas que potencian su transmisión. Además, asocian las características de la radio con las tradición oral: "La radio: es palabra hablada, es narrativa, quizás por eso nos gusta tanto en América Latina, especialmente a los pueblos indígenas y a los campesinos" (Fuentemayor y Antepaz, 2009, p. 21).

En Bolivia, Laime (2009) describe la contribución de la radiodifusión boliviana transterritorial en el fortalecimiento del pueblo Aymara desde la percepción de las propias organizaciones indígenas; para su estudio toma dos emisoras de la ciudad de La Paz, pero que tienen alcance a otras ciudades. Esta investigación muestra cómo la radio puede ayudar en la difusión del la identidad de este pueblo, sobre todo porque difunde y posibilita el aprendizaje de su lengua, de tal manera que pueda ser conservada en las prácticas de sus hablantes. Lo anterior es analizado en el marco del derecho de la autodeterminación de los pueblos.

Según parece, Bolivia es el país con más experiencias de radio indígenas en el continente, pues en el año 2007 creó el Sistema Nacional de Radios de los Pueblos Originarios conformado por 30 emisoras de radio en todo el país (Fuentemayor y Antepaz, 2009).

Recientemente en México, Castells-Talens (2011) publicó un artículo donde analiza la dinámica de las radios indígenas (denominadas indigenistas) en este país ${ }^{2}$. De manera importante, este autor discute cómo las políticas gubernamentales tienen en limbo la denominación de estas emiso-

2 De 1979 a 2000 el Estado México instaló 21 radiodifusoras, estas transmitían en lenguas indígenas y eran una herramienta de difusión cultural del Instituto Nacional Indigenista (INI). ras, pues legal y conceptualmente no las clasifican ni en emisoras indígenas ni comunitarias:

$\mathrm{Ni}$ a las organizaciones de base indígenas ni al gobierno les ha gustado nunca decir que las radios eran indígenas, sino que eran indigenistas. A los primeros porque no les querían dar "legitimidad indígena" a unas difusoras que le pertenecen al gobierno federal. A los segundos, porque querían dejar claro quiénes eran los dueños para no dejar de serlo (...) Por otro lado, ni unos ni otros parecen querer tratar estas radios como "comunitarias" por razones parecidas a las mencionadas para el término "indígena". Las organizaciones indígenas no quieren que el concepto de radio comunitaria se aplique a una radio de estructura vertical y controlada por el Estado. El gobierno tampoco quiere que sus radios reciban la etiqueta "comunitaria", por el temor de que se sugiera que la gestión tenga que ser asamblearia y la propiedad, comunitaria. (Castells- Talens, 2011, p. 131)

Sobre este tema también se puede consultar el trabajo de Chan Concha (2000), que aborda el tema de la radio indígena en el marco de la política indigenista. Además, en México se destaca el trabajo de Ramos (2005) que, más allá de estos problemas de denominación y legalidad, analiza cómo las emisoras indígenas ofrecen factores que permiten la cohesión y el fortalecimiento de las culturas de estos pueblos.

Ramos y Díez (2004) hacen una indagación sobre el uso de avisos en las radios indigenistas, de lo cual sintetizan que, en la comunidad, "los avisos permiten conocer eventos y situaciones que ocurren a integrantes de la propia comunidad y propician flujos de intercambio de información antes inexistentes. Por ejemplo, cuando se escucha algún mensaje dirigido a un vecino o pariente de la propia comunidad y se retransmite oralmente al interior de ella" (Ramos y Díez, 2004, p.185).

3 Sin embargo, no existe, según el autor, una clara diferencia entre radio indigenista y comunitaria en sus formas: ambas fomenta la participación, procuran contribuir a los problemas locales, no procuran los intereses comerciales, etc. 
También en México, Ramos (2009) analiza la inequidad mediática y multiculturalidad en la radio oficial del Estado de Puebla, profundizando en los alcances y límites de la participación indígena de la emisora: "a pesar de que estas emisoras cubren amplias regiones indígenas y dan cierta cabida a las lenguas locales, en menor o mayor medida reproducen patrones de inequidad presentes históricamente en la relación del estado con los pueblos indígenas" (Ramos, 2009, p.3). Él concluye que la emisora, cuya naturaleza es justamente posibilitar el intercambio cultural, como es la posibilidad de transmitir en lenguas indígenas, presenta desequilibrios que refuerzan el carácter hegemónico del idioma español.

\section{Radio indígena en Colombia}

En Colombia, el trabajo de Uribe-Jongbloed y Peña (2008) aborda el tema de los idiomas autóctonos minoritarios en la radio. Ellos afirman que las comunidades indígenas y raizales, dadas sus prácticas culturales cimentadas en la tradición oral, han encontrado en la radio una principal herramienta mediática, a lo que se suma el bajo costo del medio. Los autores hacen énfasis en decir que la producción radial en lenguas indígenas permite su conservación. Sin embargo, en los hallazgos de la indagación descubren que el uso del lenguaje es poco en las emisoras que analizan: "El desconocimiento del idioma por parte del equipo de trabajo de las radios indica que inscriben al medio en el área de prestigio exclusiva del español. Esto es una clara muestra de cómo este medio, en vez de convertirse en un nuevo dominio para el idioma minoritario, ayuda a aumentar el prestigio del idioma mayoritario y, de este modo, a incrementar el peligro de extinción de la lengua" (UribeJongbloed y Peña, 2008, p. 363). En ese orden de ideas concluyen: "Aunque esta investigación no encuestó todas las emisoras radiales en lengua indígena, sí evidencia los problemas de estas emisoras y plantea la necesidad de capacitar a sus directores para que establezcan un plan lingüístico y que asuman la tarea de dar mayor cabida al idioma en su programación” (p.365). Además, en su investigación, muestran emisoras que tienen problemas de orden técnico, por ejemplo, que el fluido eléctrico se interrumpe constantemente en la zona donde se ubican las emisoras.

Es pertinente citar el trabajo de González y Rodríguez (2006) sobre el ejercicio de la ciudadanía desde una emisora comunitaria en el municipio de Belén de los Andaquíes, en Caquetá, pues ellos aseguran que el sur del país, donde se ubican gran parte de las comunidades originarias, ha sido visto tradicionalmente

(...) como una bestia salvaje que necesita ser civilizada, controlada, domesticada, o - en la forma más infame del desconocimiento- fumigada desde el aire. En 1996, durante las marchas cocaleras, desde Bogotá las figuras políticas apoyadas en los grandes medios de comunicación nos vendieron a todos una imagen del sur como marginal y salvaje. Desde esta versión, era urgente que el estado central re-colonizara al Caquetá y al Putumayo; que se llevara allí, de una vez por todas, la civilización y la cultura (González y Rodríguez, 2006, p. 19).

González y Rodríguez (2006) también afirman que la emisora que ellos analizan tiene el propósito de que la región aprenda a mirar-se. "Así, Radio Andaquí va entretejiendo la tecnología de la radio con las cotidianidades de la gente, con el fin de que la gente comience a mirar su entorno como una criatura con su propia identidad, sus propios lenguajes, sus culturas híbridas resultantes del encuentro de diferentes culturas que han venido trayendo los colonos" (González y Rodríguez, 2006, p. 20).

Son pertinentes las referencias a estas citas porque, en primer lugar, se acercan a mostrar una dinámica colonial aún presente (Quijano, 2000), que afecta los imaginarios que se tienen sobre las regiones del país; por otro lado, porque ese uso de la radio para hacer reflexiones auto-reflexivas de las mismas comunidades es un rasgo que se 
puede tomar como criterio característico de las emisoras comunitarias.

Villegas y otros (2007) dan cuenta del pensamiento de las comunidades indígenas del Cauca. Ellas observan que las mingas son reuniones que posibilitan la comunicación dentro de las comunidades y que, para hacer comunicación externa a su cultura, utilizan la radio. Igualmente, explican los colectivos de estas emisoras están asociadas en la red $\mathrm{AMCIC}^{4}$, en la cual "convergen las autoridades propias, los mayores y los jóvenes, con el fin de producir programas culturales que fortalezcan la identidad y la cosmovisión" (Villegas y otros, 2007, p. 135).

Un importante antecedente es el trabajo de Rodríguez y El'Gazi (2007), quienes, bajo el título La poética de la radio indígena en Colombia, hacen un valioso análisis que aporta a la discusión del tema. Ellas plantean, de manera categórica, que "La radio indígena colombiana sólo puede ser entendida como el producto de complejas relaciones entre movimientos sociales indígenas, el conflicto armado, el Estado central y el activismo mediático" (Rodríguez y El'Gazi, 2007, p. 240).

Al igual que Castells-Talens (2011) en México, Rodríguez y El'Gazi (2007) discuten sobre las tensiones legales por la denominación de las emisoras indígenas:

En Colombia las emisoras indígenas de radio obtuvieron sus licencias como 'radios de interés público', una categoría legal reservada hasta entonces para aquellas licencias de radio asignadas a entidades públicas como gobiernos municipales o universidades públicas. Los pueblos indígenas habían solicitado un marco legal diferente específicamente diseñado para regular las emisoras indígenas de radio; los pueblos indígenas argüían que sus licencias de radio deberían ser asignadas a autoridades indígenas como 'radios indígenas', no como 'radios de interés

4 Asociación de Medios Indígenas de Colombia público' ni tampoco como 'radios comunitarias'. (Rodríguez y El'Gazi, 2007, p. 244)

Rodríguez y El'Gazi (2007) explican que los pueblos indígenas esperaban evitar la categoría de radio comunitaria porque restringe la potencia de los transmisores y, por lo tanto, limita la cobertura territorial. Las licencias para las emisoras son asignadas a las autoridades legales indígenas reconocidas por el gobierno. "Sin embargo, esta categoría impide a los pueblos indígenas financiar sus emisoras a través de anuncios locales, forzándolos a depender de subvenciones y patrocinios. Adicionalmente, como en la radio comunitaria, la legislación colombiana no permite que las emisoras indígenas de radio transmitan en red" (Rodríguez y El'Gazi, 2007, p. 244).

En el 2009, la Organización Nacional Indígena de Colombia (ONIC) publicó un diagnóstico sobre las emisoras indígenas con el apoyo del Ministerio de Cultura. En primer lugar, el informe dice que existen 25 radios indígenas bajo la licencia de interés público y 3 radios indígenas bajo la licencia de comunitarias. Estas radios están ubicadas en 12 de los 32 departamentos del país. Cauca, con 9 y Nariño, con 5, son los departamentos con más emisoras. Esto se relaciona directamente con la organización de los pueblos indígenas de esta región y sus luchas políticas. En el Cauca, además, se ubica el único centro de producción radial indígena, donde se graba, por ejemplo, música de los pueblos originarios.

En el diagnóstico se anuncia, como lo hicieron otros de los investigadores citados, los problemas económicos y técnicos de estos medios, por citar algunos: se dice que la mayoría de los programas son en vivo porque no existe forma de pre-grabar para emitir después; el gobierno les asignó, a algunas, una licencia con potencia para cubrir un buen territorio, pero el equipo que les dan no da para lograrlo; más del 70\%, para la fecha (2009), no tenían acceso a Internet, mas la comunidad buscaba la forma de acceder a la red para conseguir información e intercambiar con otras emisoras. 
De manera preponderante, para los propósitos de este artículo, el informe de la ONIC asegura que la radio indígena imita los formatos clásicos de la radio comercial, lo cual es reforzado por las capacitaciones en producción que han recibido; no obstante, existen experiencias de la formulación de formatos de expresión propios de las culturas indígenas. Por ello, el informe hace un llamado: "Dado que la radio es pura oralidad y la tradición indígena tiene una inmensa herencia oral, urge explorar otras formas distintas para acoplar el lenguaje radiofónico propio, sin perder su esencia y la cultura" (ONIC, 2009, p. 25). De la programación, los temas más reiterados tratan sobre el movimiento indígena, con subtemas como: política, identidad, derechos humanos, lengua materna, rituales, etnocultura, historia, legislación, medicina tradicional, mujer, valores y principios ancestrales, medioambiente y educación.

Las relaciones indígenas-radios-gobierno tienen un referente en el Programa Comunidad, creado en 1999 como un proyecto del Fondo de Comunicaciones del entonces Ministerio de Comunicaciones, el Ministerio de Cultura y el Fondo Financiero de Proyectos de Desarrollo-FONADE. Su propósito era apoyar el fortalecimiento, recuperación y conservación de la cultura, las tradiciones, lengua y planes de vida de los pueblos indígenas, proveyendo infraestructura de radiodifusión sonora de interés público (Ministerio de las Tecnologías de la Información y las Comunicaciones, 2010) $)^{5}$. En el 2000, la Unidad de Radio del Ministerio de Cultura de Colombia publicó las memorias del Encuentro Internacional de Radios Indígenas de América, la publicación también contiene una guía para la construcción y gestión de emisoras por parte de los pueblos originarios. Se puede ver en estos referentes un interés explícito desde políticas de Estado de incentivar y fortalecer las emisoras indígenas, sin embargo,

5 Sobre este proyecto se puede ver más información en Ministerio de Cultura (2002), los resultados parciales 2004-2005 en Ministerio de Comunicaciones (2005, pp. 59-64) y Ministerio de Comunicaciones (2007). cono se pudo observar, estas siguen padeciendo de ciertas carencias.

\section{Marcos legales de la radio indígena colombiana}

En cuanto a los referentes legales de las emisoras indígenas6, se puede empezar citando el Decreto 2001 de 1988, que designa a los cabildos indígenas como entidades públicas especiales. Más adelante, en 1991, el artículo 286 de la Constitución Política reconoce los territorios indígenas como entidades territoriales. Estos dos antecedentes son fundamentales, dado que las emisoras asignadas a los indígenas como de interés público dependen de reconocer las autoridades indígenas como públicas.

La Ley 335 de 1996, en su artículo 20, parágrafo 2, determina que el Estado debe garantizar para los grupos étnicos el acceso al uso del espectro electromagnético, los servicios públicos de telecomunicaciones y los medios masivos de comunicación, así como la creación de sus propios medios de comunicación. La Ley 397 de 1997, llamada Ley de Cultura, especifica en su artículo 13 que el Estado debe garantizar los derechos de autoría colectiva de los grupos étnicos y apoyar los procesos de etnoeducación y estimular la difusión de su patrimonio a través de los medios de comunicación, con el fin de proteger lenguas, tradiciones, usos y costumbres y saberes. Aunque no aplica para las emisoras indígenas dispuestas como de interés público, es importante citar la Resolución 719 de 2000 del Ministerio de Cultura, que denomina a los medios comunitarios o ciudadanos como expresión cultural de la nacionalidad y que poseen dinámicas propias.

6 Como marco legal internacional hay que referenciar la Declaración de las Naciones Unidas sobre los derechos de los pueblos indígenas del año 2007, que establece en su artículo 16 el derecho de estos pueblos para establecer sus propios medios y acceder a los medios de información no indígenas. Por otro lado, los estados están obligados a adoptar medidas para que los medios reflejen la diversidad cultura indígena. 
Como se observa, en las anteriores legislaciones ya emerge el tema de la comunicación para los grupos indígenas. Puntualmente, el marco legal de la radio indígena tiene su antecedente en el Decreto 1446 del 30 de agosto de 1995. Si bien este no explicita la existencia de las radios para los pueblos originarios, sí establece la primera clasificación del servicio de Radiodifusión Sonora. Este decreto, en su artículo 3, establece tres tipos de emisoras: radiodifusión comercial, radiodifusión de interés público, radiodifusión comunitaria. Las emisoras indígenas están en el segundo tipo.

La anterior legislación fue actualizada con el Decreto 2805 DE 2008, que define puntualmente esta clasificación así: a) Radiodifusión sonora comercial: cuando la programación del servicio está destinada a la satisfacción de los hábitos y gustos del oyente y el servicio se presta con ánimo de lucro, sin excluir el propósito educativo, recreativo, cultural, científico e informativo que orienta el Servicio de Radiodifusión Sonora en general; b) Radiodifusión sonora de interés público: cuando la programación se orienta a satisfacer necesidades de comunicación del Estado con los ciudadanos y comunidades, la defensa de los derechos constitucionales, la protección del patrimonio cultural y natural de la nación, a fin de procurar el bienestar general y el mejoramiento de la calidad de vida de la población, sin ánimo de lucro, a cargo y bajo la titularidad del Estado. c) Radiodifusión sonora comunitaria: cuando la programación está orientada a generar espacios de expresión, información, educación, comunicación, promoción cultural, formación, debate y concertación que conduzcan al encuentro entre las diferentes identidades sociales y expresiones culturales de la comunidad, la promoción de la democracia, la participación y los derechos fundamentales de los colombianos que aseguren una convivencia pacífica (artículo 18, Decreto 2805 DE 2008).

De manera preponderante, el Decreto 2805, en su artículo 33, determina que "Para la promoción y respeto de la diversidad cultural, las transmisiones de radiodifusión sonora también pueden transmitirse en dialectos indígenas o lenguas nativas".

Un artículo criticado por los indígenas de este decreto es el número 54, que prohíbe a las estaciones de radiodifusión comunitaria y de interés público encadenarse. Este es uno de los puntos, sumado a la posibilidad de recibir ingresos por pautas, por los cuales los movimientos indígenas exigen un marco jurídico especial para las radios indígenas.

\section{Conclusiones y recomendaciones}

Después de la anterior revisión se puede decir que la radio indígena en Colombia ha tenido, desde el año 2000, un significativo crecimiento, dado que los pueblos originarios han encontrado en ella un medio que satisface sus necesidades mediáticas y congenia con su tradición oral; además, el gobierno ha apoyado esta iniciativa. Sin embargo, como se pudo ver en los primeros apartados del artículo, las investigaciones sobre radio en general y radio indígena en particular son escasas en el país.

Existe una tensión legal en la eclosión de las radios indígenas en Colombia categorizadas como de interés público, pues las comunidades exigen una legislación especial que les permita la radiodifusión en cadena, posibilidad que tiene una inmanencia política que permitiría al movimiento indígena una importante plataforma mediática, y recibir dinero por pauta publicitaria. Como se puede ver, estas exigencias implican una categoría nueva a las determinadas en el Decreto 2805 de 2008, pues combina intereses comerciales, tanto públicos como comunitarios.

En Colombia y en el continente, las investigaciones sobre radio indígena han dado cuenta del uso del medio para conservar y difundir la cultura de los pueblos, pero señalan la supremacía del 
español sobre las lenguas originarias en la producción radiofónica. Asimismo, enumeran problemas técnicos y económicos en estas emisoras. Si bien las investigaciones han mostrado la importancia de la radio en la divulgación cultural, hace falta observar cómo la cosmovisión de cada pueblo re-significa el lenguaje radiofónico y los géneros y formatos comerciales.

Se propone, siguiendo esta línea, incursionar en investigaciones que observen cómo el lenguaje radiofónico permite representar y poner en común la cosmovisión de los pueblos indígenas, cómo lo radiofónico, que tiene un lenguaje estructurado y estipulado desde la modernidad occidental, es re-significado y re-articulado desde la cosmovisión particular y las prácticas culturales de los pueblos indígenas (una suerte de transmodernidad propuesta por Dussel, 2005). La cosmovisión, en este caso, podría estar enmarcada en la Ley de Origen que siguen los pueblos colombianos.

\section{Referencias bibliográficas}

Albó, X. (1998). Expresión indígena, diglosia y medios de comunicación. En Lopez, L. y Jung, I. (Comp.). Sobre las huellas de la voz. Madrid: Ediciones Morata.

Alí, Marizio. (2011). Medios de comunicación, asuntos étnicos e intercultura en Colombia. En Razón y Palabra. N. 74. Recuperado de http://www.razonypalabra.org.mx/N/N74/VARIA74/23AliV74.pdf

Arteaga, M. y González, J. (2004). Memoria para periodistas. "Es posible otra mirada". Medios de comunicación e identidades indígenas. Cali: Universidad del Valle

Antequera, J. Obregón, R. (2002). La radio como dinamizadora de procesos sociales y culturales en barranquilla. En: Investigación y desarrollo. V. 10 N. 2. Universidad del norte. pp. 146-69.

Barrios (2011). De la onda a la web. Paralelo entre la radio convencional y la radio virtual. Bogotá: Universidad Jorge Tadeo Lozano.
Bennett, N. (2004). La Red Rusa de Información Rural. En Bruce, G. (Ed.). Secreto a voces Radio, NTICs e interactividad. Roma Organización de las Naciones Unidas para la Agricultura y la Alimentación.

Castellanos, N. (2001). La radio colombiana, una historia de amor y de olvido. En Signo y Pensamiento. V. X. N. 39. pp. 15-23

Castells-Talens, A. (2011). ¿Ni indígena ni comunitaria? La radio indigenista en tiempos neoindigenistas. En Comunicación y sociedad. No. 15. pp 123-142.

Chan Concha, L. (2000). La radio indígena en el marco de la política indigenista. (Tesis de licenciatura). México: UNAM.

Cebrián, M. (2007). Modelos de radio, desarrollos e innovaciones: del diálogo y participación a la interactividad. Madrid: Fragua.

Dussel, E. (2005). Transmodernidad e interculturalidad. Recuperado de http://www.afyl.org/transmodernidadeinterculturalidad.pdf

Fuentemayor, M. y Antepaz, O. (2009). En: II Congreso Invecom. Recuperado de http://congresoinvecom. org/index.php/invecom2009/invecom2009/paper/ view/46/51

Gil, F. (1992). Influencia política y poder económico en los medios de comunicación: las cadenas radiofónicas colombianas. En Revista CIDOB D'Afers Internacionals. N. 23-24. Pp. 225-254.

González, J. M. (2009). Estudio de audiencias a estudiantes de la Pontificia universidad Javeriana en relación a la emisora Javeriana Estéreo. Bogotá: Pontificia Universidad Javeriana.

González, J. y Arteaga, M. (2005). La representación de lo indígena en los medios de comunicación. Medellín: ASCUN - Hombre Nuevo Editores.

González, A. y Rodríguez, C. (2006) Alas para tu voz. Ejercicios de ciudadanía desde una emisora comunitaria. En: Rodríguez, C. (Ed.) Lo que le vamos quitando a la guerra. Bogotá: Centro de Competencia en Comunicación para América Latina. Recuperado de http://www.c3fes.net/docs/andaqui.pdf 
Gumucio, A. (2004). El cuarto mosquetero: la comunicación para el cambio social. En Revista Investigación y Desarrollo. Vo. 12 No. 1. pp. 2-23.

Herrera, S. (2007). Las nuevas modalidades para la participación de los jóvenes en la radio española. Revista Trípodos. $N^{\circ}$ 20. Barcelona: Universidad Ramón Llull pp. 171-188.

Ilboudo, J. y Castello, R. (2004). Construir puentes para cerrar la brecha digital rural. Vincular la radio rural y las NTICs en África. En Bruce, G. (Ed.). Secreto a voces Radio, NTICs e interactividad. Roma Organización de las Naciones Unidas para la Agricultura y la Alimentación.

Iriarte, M. (2006). Comunicación para el cambio social: la radio indígena comunitaria Pataxó-Hã-hã-hãe en Brasil. En Moneta, C. (Ed.). Jardín de senderos que se encuentran: Políticas públicas y diversidad cultural en el MERCOSUR. (pp. 117-128). Montevideo: UNESCO.

Laime, F. (2009). Radiodifusión boliviana aymara en el fortalecimiento del pueblo aymara. Más allá de las frontera. (Tesis de maestría). Cochabamba: Mayor de San Simón

Lalinde, A. (1992). Radio y cultura profesional. La producción de noticias en Caracol Radio. Bogotá: Universidad Javeriana-Colciencias. Informe final de investigación.

Lalinde, A. (1999). Una Mirada al género desde la radio. En Gaceta, N. 44-45, p. 50.

Lalinde, A. (1998). Radio Informativa ¿Es posible la participación? En Signo y Pensamiento, N. 33.

Lara, Ariel y Vera, Cesar. (2003). La radio en la era digital. [Tesis] Santiago: Instituto de la Comunicación y la Imagen. Universidad de Chile. Recuperado de: http://www.archi.cl/nuevo/images/biblio/8_ RADIO _ EN _ LA _ ERA _ DIGITAL _ _ SEMINA$\mathrm{RIO}$ _ GRADO _ U _ CHILE.pdf

López de la Roche. (1999). Las relaciones entre comunicación y cultura y la formulación de políticas culturales. En Seminario Distrital Políticas Culturales para Santafé de Bogotá. Bogotá: Fondo Mixto para la Promoción de la Cultura y las Artes de Santafé de Bogotá. Arte Siglo XXI Imago. Ministerio de Cultura.
Ministerio de las Tecnologías de la Información y las Comunicaciones. (2010). Programa Comunidad. Recuperado de http://archivo.mintic.gov.co/mincom/ faces/index.jsp?id=10769

MINGA. (2005). La representación de lo indígena en los medios de comunicación. Santiago de Cali: S.E. Recuperado de http://www.colombiaaprende.edu. $\mathrm{co} / \mathrm{html} /$ investigadores/1609/articles-81566 _ archivo.pdf

Múnera, L. (1999). La radio y la televisión en Colombia. Bogotá: APRA.

Ministerio de Comunicaciones. (2002). Diagnóstico del servicio comunitario de radiodifusión sonora en Colombia. Bogotá: Ministerio de Comunicaciones de Colombia.

Ministerio de Comunicaciones. (2005). Informe de gestión sector de las telecomunicaciones julio 2004 - julio 2005. Bogotá: República de Colombia. Recuperado de http://www.lib.utexas.edu/benson/lagovdocs/ colombia/federal/comunicaciones/Inf-gestiontelecomunica-2004-2005.pdf

Ministerio de Comunicaciones. (2007). Programa Comunidad - Señal de Cultura y Diversidad: Emisoras de Interés Público para Pueblos Indígenas. Documento Interno: Programa Comunidad.

Ministerio de Cultura. (2002) Proyecto Comunidad: Señal de Cultura y Diversidad. Programa de Emisoras para Pueblos Indígenas (Project Report). Bogotá: Ministerio de Cultura.

ONIC. (2009). Diagnóstico integral de emisoras y/o radios indígenas. Bogotá: Ministerio de Cultura y ONIC. Recuperado de http://archivo.mintic.gov.co/mincom/documents/portal/documents/root/mfardila/ docs/DiagnosticoEmisorasIndigenas.pdf

Otero, J. (2007). La representación de lo indígena en los medios de comunicación. Recuperado de http:// www.c3fes.net/docs/representacionindigena.pdf

Otero, J. (2008). El derecho a la comunicación en el plan de vida de los pueblos indígenas del Cauca. S.L: Centro de Competencia en Comunicación para 
América Latina. Recuperado de http://www.c3fes. net/docs/comunicacionindigenacaucacric.pdf

Pareja, R. (1984). Historia de la radio en Colombia 1929 - 1980. Bogotá: Servicio Colombiano de Comunicación Social.

Pérez, G. (1998). La radio del tercer milenio. Bogotá: Caracol Radio.

Quijano, Aníbal (2000) Colonialidad del poder, eurocentrismo y América Latina. En Lander, E. (comp.). La colonialidad del saber: eurocentrismo y ciencias sociales. Perspectivas Latinoamérica. Buenos Aires: Consejo Latinoamericano de Ciencias Sociales.

Ramos, J. (2005). Ecos de "La voz de la montaña": la radio como factor de cohesión y fortalecimiento cultural de los pueblos indígenas. (Tesis de doctorado). México: UNAM.

Ramos, J. y Díez, A. (2004). Viejas y nuevas tecnologías. $\mathrm{El}$ servicio de avisos de las radios indigenistas de México. En: Bruce, G. (Ed.). Secreto a voces. Radio, NTICs e interactividad. Roma: Organización de las Naciones Unidas para la Agricultura y la Alimentación.

Ramos, J. (2009). Inequidad mediática y multiculturalidad: Alcances y límites de la participación indígena en la radio oficial del estado de Puebla, México. En Prepared for delivery at the 2009 Congress of the Latin American Studies Association, Rio de Janeiro, Brazil. June 11-14, 2009. Recuperado de http://lasa. international.pitt.edu/members/congress-papers/ lasa2009/files/RamosRodriguezJose.pdf

Rocha, C., Aldana, Y., Rodríguez, L. y Ortiz, G. (2010). La radio comunitaria en Cundinamarca: una posibilidad para pensar el desarrollo. Bogotá: Corporación universitaria minuto de dios. Facultad de Ciencias de la Comunicación.

Rodríguez, C. y El'Gazi, J. (2007). La poética de la radio indígena en Colombia. En Rincón y otros. Ya no es posible el silencio. Bogotá: Centro de Competencia en Comunicación para América Latina - Friedrich Ebert Stiftung

Rodríguez, E. (2011). Nativos digitales en la reconfiguración radiofónica. Revista Razón y Palabra N. 76.
En línea: http://www.razonypalabra.org.mx/N/N76/ varia/3a\%20entrega/32 _ Rodriguez _ V76.pdf

Rojas, S. (2009). Medios de comunicación y políticas públicas: producción de conocimiento en Colombia. En Comunicación y Ciudadanía. N. 2. pp. 104-113

Ruiz, D. (2009). La radio como puesta en escena de las creencias en Bogotá. Bogotá: Pontificia Universidad Javeriana.

Saffon, M. y Uprimny, R. (2007). En búsqueda de una voz en la radio. En Rincón y otros. Ya no es posible el silencio. Bogotá: Centro de Competencia en Comunicación para América Latina - Friedrich Ebert Stiftung

Salazar, J. (2002). Activismo indígena en América Latina: Estrategias para una construcción cultural de las tecnologías de información y comunicación. En Journal of Iberian and Latin American Studies. V.8 N.2. pp. 61-79 Recuperado de http://uws.academia. edu/JuanFranciscoSalazar/Papers/802400/Activismo _ indigena _ en _ America _ Latina _ Estrategias _ para _ una _ construccion _ cultural _ de _las_tecnologias _ de _ informacion _ y _ comunicacion

Téllez, H. (1974). Cincuenta años de la radiodifusión colombiana. Medellín: Editorial Bedout.

Unidad de Radio. (2000) Radios y Pueblos Indígenas. Memorias del Encuentro Internacional de Radios Indígenas de América. Bogotá: Ministerio de Cultura.

Urdaneta, M. (1994). Historia de la radiodifusión en Barranquilla. Barranquilla: Editorial Uniautónoma.

Uribe-Jongbloed, E. y Peña, M. (2008). Medios en idiomas autóctonos minoritarios en Colombia. El caso de la radio. En Palabra Clave. V. 11 N. 2. pp. 355-366

Valderrama, C. (2009). La investigación en medios de comunicación en Colombia (1980-2009). En Revista Nómadas. No. 31. pp. 262-276

Valderrama H. y Rojas C. (2007). Estado del arte sobre la investigación en medios en Colombia 1980- 2005. En Revista Nómadas. No. 26. pp. 234-236 
Villegas, A., Ramírez, N., Tunubalá, J. Martínez, M. y Andrade, M. (2007). Palabra, semilla de convivencia [Mingas de pensamiento, Comunidades indígenas del Cauca]. En Rincón y otros. Ya no es posible el silencio. Bogotá: Centro de Competencia en Comunicación para América Latina - Friedrich Ebert Stiftung
Winocur, R. (2002). Ciudadanos mediáticos: la construcción de lo público en la radio. Barcelona: Gedisa. 